\title{
Exclusive processes in ep collisions at the EIC and LHeC: A closer look on the predictions of saturation models
}

\author{
Ya-Ping Xie $\oplus^{1,2, *}$ and V.P. Gonçalves $\oplus^{3,1, \uparrow}$ \\ ${ }^{1}$ Institute of Modern Physics, Chinese Academy of Sciences, Lanzhou 730000, China \\ ${ }^{2}$ University of Chinese Academy of Sciences, Beijing 100049, China \\ ${ }^{3}$ High and Medium Energy Group, Instituto de Física e Matemática, Universidade Federal de Pelotas, \\ Caixa Postal 354, CEP 96010-900, Pelotas, Rio Grande do Sul, Brazil
}

(Received 8 December 2021; accepted 6 January 2022; published 31 January 2022)

\begin{abstract}
The exclusive production of vector mesons and photons in $e p$ collisions is investigated considering three phenomenological saturation models based on distinct assumptions for the treatment of the dipolehadron scattering amplitude. The latest high precision HERA data for the reduced and vector meson cross sections are used to update the saturation model proposed by Marquet Peschanski, and Soyez, which predicts that the saturation scale is dependent of the squared momentum transfer $t$. The MarquetPeschanski-Soyez predictions for the photon virtuality, energy and $t$ dependencies of the exclusive $\rho, J / \Psi$, and deeply virtual Compton scattering cross sections are presented and a detailed comparison with the results derived using the impact parameter saturation models is performed. Our results indicate that a future experimental analysis of the $t$ distribution $d \sigma / d t$ for exclusive processes in the kinematical range that will covered by the EIC and $\mathrm{LHeC}$, considering the distinct photon polarizations and large values of $t$, will be able to discriminate between the distinct approaches for the QCD dynamics at high energies.
\end{abstract}

DOI: 10.1103/PhysRevD.105.014033

\section{INTRODUCTION}

The main goal of the future Electron-Ion Colliders at the BNL (EIC) [1] and at the LHC (LHeC) [2] is to achieve a deeper knowledge of the hadronic structure at high energies through the deep inelastic scattering process, where an electron emits a virtual photon that interacts with a proton/nuclear target. In particular, the proton structure can then be studied through the $\gamma^{*} p$ interaction, with the behavior of the observables being determined by the QCD dynamics at high energies. Previous experimental studies, carried out at HERA, have shown that the gluon density inside the proton grows with the energy (see, e.g., Ref. [3]). Therefore, at high energies, a hadron becomes a dense system and the nonlinear (saturation) effects inherent to the QCD dynamics may become visible [4]. This aspect has motivated the development of an intense phenomenology over the last years, which has demonstrated that diffractive processes, characterized by a rapidity gap in the final state,

\footnotetext{
*xieyaping@impcas.ac.cn

tbarros@ufpel.edu.br
}

Published by the American Physical Society under the terms of the Creative Commons Attribution 4.0 International license. Further distribution of this work must maintain attribution to the author(s) and the published article's title, journal citation, and DOI. Funded by SCOAP. are the most promising one to study the high-density regime of QCD and to probe the gluonic structure of protons and nuclei (see, e.g., Refs. [5-27]). In particular, the exclusive production of a vector meson or a photon, represented in Fig. 1, was demonstrated to be strongly sensitive to the underlying QCD dynamics, since this process is driven by the gluon content of the target, with the cross section being proportional to the square of the dipole-hadron scattering amplitude. Moreover, by measuring the squared momentum transfer $t=-\boldsymbol{q}^{2}$, where $\boldsymbol{q}$ is the momentum transfer, the transverse spatial distribution of the gluons in the hadron wave function can also be studied [28].

The exclusive processes in $e p$ collisions are characterized by the produced state $E(=\rho, J / \Psi, \gamma)$ and an intact proton in the final state, with a rapidity gap separating these systems. In the color dipole formalism [29], the scattering amplitude can be factorized in terms of the photon wave function $\Psi^{\gamma}$, which describes the fluctuation of the virtual photon into a $q \bar{q}$ color dipole, the dipole-hadron scattering amplitude $\mathcal{T}$, which describes the scattering by a color singlet exchange $(I P)$, and the wave function $\Psi^{E}$ that describes the $q \bar{q}$ recombination into the exclusive final state $E$. The dipole-hadron scattering amplitude $\mathcal{T}$ can be expressed in terms of the momentum transfer $\boldsymbol{q}$ or the impact parameter $\boldsymbol{b}$, with both representations being related by a Fourier transform. 


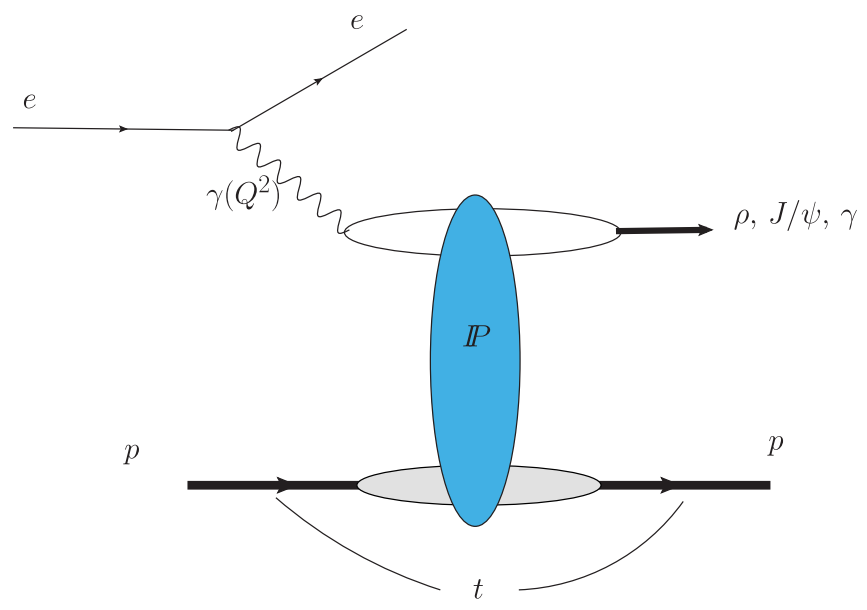

FIG. 1. Exclusive processes in $e p$ collisions.

In recent years, several groups [30-34] have focused on the impact parameter representation and proposed different phenomenological approaches to describe $\mathcal{T}(x, \boldsymbol{r}, \boldsymbol{b})$, which are based on the color glass condensate (CGC) formalism [35] and that successfully describe a large set of observables in $e p, p p, p A$, and $A A$ collisions. In particular, in our analysis we will consider the impact parameter saturation model (BSAT) and impact parameter color glass condensate model (BCGC) approaches [31,32], which are able to describe the data for exclusive processes in the kinematical range covered by the HERA experiment. A shortcoming of BSAT and BCGC models is that the $\boldsymbol{b}$ dependencies of the dipole amplitudes are ad hoc assumptions of the models, which are expected to capture the main contributions associated to nonperturbative physics that are not taken into account by the CGC weak-coupling approach. Such aspect has motivated an intense debate in the literature (see, e.g., Refs. [34,36]). In contrast, the modeling of the scattering amplitude in the momentum transfer representation, $\mathcal{T}(x, \boldsymbol{r}, \boldsymbol{q})$, was only discussed in Ref. [37] considering the results derived in Refs. [38,39] for the exact solutions of the Balitsky-Kovchegov (BK) equation [40] at nonzero momentum transfer. The Marquet-Peschanski-Soyez model, denoted as MPS hereafter, predicts that the saturation scale becomes proportional to $t$ and that nonperturbative contributions can be factorized when the scattering amplitude is described in the momentum transfer representation, which is not the case in the BSAT and BCGC models. One of the goals of this paper is to update the analysis performed in Ref. [37] considering the latest combined HERA data, which were already used to update the BSAT and BCGC models. Another goal is to perform a detailed comparison between the predictions of the MPS, BSAT, and BCGC models for the exclusive $\rho, J / \Psi$, and $\gamma$ production in $e p$ collisions at the kinematical range that will be probed by the EIC and LHeC. In particular, we will present an extensive comparison between the predictions for the exclusive cross sections considering distinct values of the photon virtuality $Q^{2}$, center-of-mass energy $W$ and squared momentum transfer $t$. Our main motivation is to verify if the analysis of exclusive processes in future $e p$ colliders could be used to discriminate between these distinct treatments of the QCD dynamics at high energies.

It is important to emphasize that, in recent years, several groups have improved the treatment of exclusive processes in the dipole approach by estimating higher order corrections for the photon impact factor and/or improving the description of the vector meson wave function (see, e.g., Refs. [41-43]). However, the proper inclusion of the impact-parameter dependence of collisions and nonforward corrections in the CGC approach at next to leading order is still a theme of debate, which motivates the analysis of the existing phenomenological models performed in this paper.

The paper is organized as follows. In the next section, we present a brief overview of the formalism needed for the description of the exclusive processes in $e p$ collisions and discuss the distinct models for the dipole-proton scattering amplitude employed in our analysis. In Sec. III, we exhibit the results for the updated version of the MPS model, obtained by fitting the latest HERA data, and its predictions are compared with those derived using the BSAT and BCGC models. Moreover, we present our predictions for the total cross section and transverse momentum distribution considering $e p$ collisions at the EIC and LHeC energies. Finally, in Sec. IV we summarize our main conclusions.

\section{FORMALISM}

In the color dipole formalism, the scattering amplitude for the $\gamma^{*} p \rightarrow E p$ process can be expressed in the momentum representation as follows [37]:

$$
\begin{aligned}
& \mathcal{A}_{T, L}^{\gamma^{*} p \rightarrow E p}\left(x, Q^{2}, t=-\boldsymbol{q}^{2}\right) \\
& \quad=i \int d^{2} \boldsymbol{r} \int \frac{d z}{4 \pi}\left(\psi_{E}^{*} \psi_{\gamma}\right)_{T, L}\left(z, \boldsymbol{r}, Q^{2}\right) e^{-i z \boldsymbol{q} \cdot \boldsymbol{r}} \mathcal{T}(x, \boldsymbol{r}, \boldsymbol{q}),
\end{aligned}
$$

where $\boldsymbol{q}$ is the momentum transfer, $Q^{2}$ is the photon virtuality, and $x=\left(Q^{2}+M^{2}\right) /\left(W^{2}+Q^{2}\right)$, with $W$ being the center-of-mass energy of the virtual photon-proton system and $M$ is the mass of the produced final state. Moreover, $r$ is the size of the $q \bar{q}$ dipole and $z$ and $(1-z)$ are the momentum fractions of the incoming photon momentum carried by the quark and antiquark, respectively. The overlap functions $\left(\psi_{E}^{*} \psi_{\gamma}\right)_{T, L}$ describe the fluctuation of a photon with transverse $(T)$ or longitudinal $(L)$ polarization into a color dipole and the subsequent formation of the final state $E$. In the case of the exclusive photon production, denoted as deeply virtual Compton scattering (DVCS), only the transverse polarization contributes, and the overlap function $\left(\psi_{\gamma}^{*} \psi_{\gamma}\right)$ can be calculated using perturbation theory, being given by [31] 


$$
\begin{aligned}
\left(\psi_{\gamma}^{*} \psi_{\gamma}\right)_{T} & =\sum_{f}\left(\psi_{\gamma}^{*} \psi_{\gamma}\right)_{T}^{f}\left(z, \boldsymbol{r}, Q^{2}\right), \\
& =\sum_{f} \frac{2 N_{c}}{\pi} \alpha_{e m} e_{f}^{2}\left\{\left[z^{2}+(1-z)^{2}\right] \epsilon K_{1}(\epsilon r) m_{f} K_{1}\left(m_{f} r\right)+m_{f}^{2} K_{0}(\epsilon r) K_{0}\left(m_{f} r\right)\right\},
\end{aligned}
$$

where $m_{f}$ and $e_{f}$ are the mass and charge of a quark with flavor $f$ and $\epsilon^{2}=z(1-z) Q^{2}+m_{f}^{2}$. In contrast, the modeling of the overlap function for the vector meson production is still a theme of debate, with different models being able to describe, e.g., the HERA data. We will assume that the vector meson is predominantly a quark-antiquark state and that its spin and polarization structures are the same as the photon [30,31,44-47]. Such assumptions imply that the transverse and longitudinal overlap functions are given by

$$
\begin{aligned}
\left(\Psi_{V}^{*} \Psi_{\gamma}\right)_{T} & =\hat{e}_{f} e \frac{N_{c}}{\pi z(1-z)}\left\{m_{f}^{2} K_{0}(\epsilon r) \phi_{T}(r, z)-\left(z^{2}+(1-z)^{2}\right) \epsilon K_{1}(\epsilon r) \partial_{r} \phi_{T}(r, z)\right\}, \\
\left(\Psi_{V}^{*} \Psi_{\gamma}\right)_{L} & =\hat{e}_{f} e \frac{N_{c}}{\pi} 2 Q z(1-z) K_{0}(\epsilon r)\left[M_{V} \phi_{L}(r, z)+\delta \frac{m_{f}^{2}-\nabla_{r}^{2}}{M_{V} z(1-z)} \phi_{L}(r, z)\right],
\end{aligned}
$$

where $\hat{e}_{f}$ is the effective charge of the quarks, $N_{c}=3$, and $\phi_{T, L}(r, z)$ define the scalar part of the vector meson wave functions. In this analysis we will consider two popular models employed in the literature- the boosted Gaussian (BG) and light cone Gauss (LCG) - which differ in the assumptions for the parameter $\delta$ and functions $\phi_{T, L}(r, z)$. In the boosted Gaussian model, $\delta=1$ and these functions are expressed as follows:

$$
\phi_{T, L}(z, r)=\mathcal{N}_{T, L} z(1-z) \exp \left(-\frac{m_{f}^{2} \mathcal{R}^{2}}{8 z(1-z)}-\frac{2 z(1-z) r^{2}}{\mathcal{R}^{2}}+\frac{m_{f}^{2} \mathcal{R}^{2}}{2}\right)
$$

where $\mathcal{N}_{T, L}$ and $\mathcal{R}$ are free parameters to be determined by the normalization condition of the wave function and by the decay width. On the other hand, in the LCG model, $\delta=0$ and the scalar functions are given by

$$
\begin{gathered}
\phi_{T}(z, r)=N_{T}[z(1-z)]^{2} \exp \left(-\frac{r^{2}}{2 R_{T}^{2}}\right), \\
\phi_{L}(z, r)=N_{L} z(1-z) \exp \left(-\frac{r^{2}}{2 R_{L}^{2}}\right) .
\end{gathered}
$$

As in the BG model, the parameters $N_{T, L}$ and $R_{T, L}$ are fixed by the normalization and decay width (see, e.g., Ref. [31] for details). The parameters adopted in our analysis are presented in Table I for distinct values of the light quark and charm masses. Some of the parameters are taken from Refs. [10,31].
The energy, photon virtuality, and transverse momentum dependencies of the scattering amplitude for exclusive processes are determined by the evolution of $\mathcal{T}$ and, consequently, are strongly dependent on the description of the QCD dynamics. At small values of $x$, the parton densities increase and the scattering amplitude approaches the unitarity limit, where nonlinear effects become important and should be taken into account [4]. In order to describe the saturation regime of QCD one can consider the BK equation [40], which resums QCD fan diagrams in the leading-logarithmic approximation. This equation has been extensively studied in the literature, mainly considering that the impact parameter dependence can be factorized, i.e.,

\begin{tabular}{|c|c|c|c|c|c|c|c|c|c|c|}
\hline \multirow[b]{2}{*}{ Vector meson } & \multirow[b]{2}{*}{$M_{V}(\mathrm{GeV})$} & \multirow[b]{2}{*}{$m_{f}(\mathrm{GeV})$} & \multirow[b]{2}{*}{$\hat{e}_{f}$} & \multicolumn{3}{|c|}{ Boosted Gaussian } & \multicolumn{4}{|c|}{ Light cone Gauss } \\
\hline & & & & $\mathcal{R}^{2}\left(\mathrm{GeV}^{-2}\right)$ & $\mathcal{N}_{L}$ & $\mathcal{N}_{T}$ & $R_{L}\left(\mathrm{GeV}^{-2}\right)$ & $R_{T}\left(\mathrm{GeV}^{-2}\right)$ & $N_{L}$ & $N_{T}$ \\
\hline$\rho$ & 0.776 & 0.01 & $1 / \sqrt{2}$ & 13.3 & 0.894 & 1.004 & 10.4 & 21.9 & 1.79 & 5.90 \\
\hline$\rho$ & 0.776 & 0.14 & $1 / \sqrt{2}$ & 12.9 & 0.853 & 0.911 & 10.4 & 21.9 & 1.79 & 4.47 \\
\hline$J / \psi$ & 3.097 & 1.27 & $2 / 3$ & 2.45 & 0.592 & 0.596 & 3.0 & 5.6 & 0.83 & 1.44 \\
\hline$J / \psi$ & 3.097 & 1.4 & $2 / 3$ & 2.3 & 0.575 & 0.578 & 3.0 & 6.5 & 0.83 & 1.23 \\
\hline
\end{tabular}
$\mathcal{T}(x, \boldsymbol{r}, \boldsymbol{b})=\mathcal{T}(x, \boldsymbol{r}) S(\boldsymbol{b})$ (see, e.g., [48]). It has been shown that the equation for $\mathcal{T}(x, r)$ is equivalent to the FisherKolmogorov-Petrovsky-Piscounov equation [49] and admits asymptotic traveling-wave solutions [50], which imply the geometric scale property, $\mathcal{T}(x, r)=\mathcal{T}\left(r Q_{s}(x)\right)$,

TABLE I. Parameters for the vector meson wave functions used in our analysis [10,31]. 
that is observed in the HERA data [51-53]. In Ref. [38] the authors demonstrated that the traveling-wave method can be extended for the nonforward case when the BK equation is expressed in the momentum space and that the geometric scaling property can be extended to the case of nonzero momentum transfer. Based on the results obtained in Refs. [38,39], the authors have proposed in Ref. [37] the phenomenological MPS model for $\mathcal{T}(x, \boldsymbol{r}, \boldsymbol{q})$, in which the scattering amplitude is expressed as follows:

$$
\mathcal{T}_{M P S}(x, \boldsymbol{r}, \boldsymbol{q})=2 \pi R_{p}^{2} f(\boldsymbol{q}) \times\left\{\begin{array}{ll}
\mathcal{N}_{0}\left(\frac{r Q_{s}(x, \boldsymbol{q})}{2}\right)^{2\left(\gamma_{s}+(1 / \kappa \lambda Y) \ln \left(2 / r Q_{s}(x, \boldsymbol{q})\right)\right)}, & r Q_{s}(x, \boldsymbol{q}) \leq 2 \\
1-\exp \left(-a \ln ^{2}\left(\operatorname{br} Q_{s}(x, \boldsymbol{q})\right)\right), & r Q_{s}(x, \boldsymbol{q})>2
\end{array},\right.
$$

where $\mathcal{N}_{0}=0.7, Y=\ln (1 / x), \kappa=9.9$ is obtained from the Balitsky-Fadin-Kuraev-Lipatov kernel, and the constants $a$ and $b$ are given by

$$
\begin{aligned}
& a=-\frac{\mathcal{N}_{0}^{2} \gamma_{s}^{2}}{\left(1-\mathcal{N}_{0}\right)^{2} \ln \left(1-\mathcal{N}_{0}\right)}, \\
& b=\frac{1}{2}\left(1-\mathcal{N}_{0}\right)^{-\left(1-\mathcal{N}_{0}\right) /\left(2 \mathcal{N}_{0} \gamma_{s}\right)} .
\end{aligned}
$$

The MPS model generalizes the forward saturation model proposed in Ref. [54] for the nonforward case and is characterized by a saturation scale that is dependent on the momentum transfer $\boldsymbol{q}$ and by the factorization of the form factor $f(\boldsymbol{q})$, which describes the momentum transfer dependence of the proton vertex. As in Ref. [37], we will assume that $Q_{s}(x, \boldsymbol{q})=\left(x / x_{0}\right)^{\lambda / 2}\left(1+c \boldsymbol{q}^{2}\right)$ and $f(\boldsymbol{q})=e^{-B q^{2}}$. The parameters $c, B, R_{p}, \gamma_{s}, \lambda$, and $x_{0}$ will be obtained by fitting the latest combined HERA data for the reduced and vector meson cross sections (see below).

As discussed in Sec. I, the scattering amplitude can also be estimated in the impact parameter space. In this space, $\mathcal{A}$ is expressed as follows:

$$
\begin{aligned}
& \mathcal{A}_{T, L}^{\gamma^{*} p \rightarrow E p}\left(x, Q^{2}, t=-\boldsymbol{q}^{2}\right) \\
& \quad=i \int d^{2} \boldsymbol{r} \int \frac{d z}{4 \pi} \int d^{2} \boldsymbol{b}\left(\psi_{E}^{*} \psi_{\gamma}\right)_{T, L} e^{-i(\boldsymbol{b}-(1-z) \boldsymbol{r}) \cdot \boldsymbol{q}} \mathcal{T}(x, \boldsymbol{r}, \boldsymbol{b}) .
\end{aligned}
$$

In our analysis we would like to compare the MPS predictions with those derived using the BSAT and BCGC models for $\mathcal{T}(x, \boldsymbol{r}, \boldsymbol{b})$, which consider distinct assumptions for the impact parameter dependence. The BSAT model assumes an eikonalized form for the dipole-proton scattering amplitude that depends on a gluon distribution evolved via the Dokshitzer-Gribov-Lipatov-Altarelli-Parisi equation, being given by [31]

$$
\begin{aligned}
& \mathcal{T}_{\mathrm{BSAT}}(x, \boldsymbol{r}, \boldsymbol{b}) \\
& \quad=2\left\{1-\exp \left[-\frac{\pi^{2}}{2 N_{c}} \boldsymbol{r}^{2} \alpha_{s}\left(\mu^{2}\right) x g\left(x, \mu^{2}\right) T_{p}(\boldsymbol{b})\right]\right\},
\end{aligned}
$$

where $T_{p}(\boldsymbol{b})=\left(1 / 2 \pi B_{p}\right) \exp \left(-\boldsymbol{b}^{2} / 2 B_{p}\right)$ is the profile function. On the other hand, in the BCGC model, the saturation scale is assumed to be dependent of the impact parameter and the dipole-proton scattering amplitude is written as [32]

$$
\begin{aligned}
& \mathcal{T}_{\mathrm{BCGC}}(x, \boldsymbol{r}, \boldsymbol{b}) \\
& =2 \times\left\{\begin{array}{ll}
\mathcal{N}_{0}\left(\frac{r Q_{s}}{2}\right)^{2\left(\gamma_{s}+(1 / \kappa \lambda Y) \ln \left(2 / r Q_{s}\right)\right)}, & r Q_{s} \leq 2 \\
1-\exp \left(-a \ln ^{2}\left(b r Q_{s}\right)\right), & r Q_{s}>2
\end{array},\right.
\end{aligned}
$$

with $Q_{s}(x, \boldsymbol{b})=\left(x / x_{0}\right)^{\lambda / 2} \exp \left(-\frac{b^{2}}{4 \gamma_{s} B_{p}}\right)$. In our analysis using the BSAT and BCGC models, we will consider the parameters determined in Refs. [55,56] using the latest HERA data and assuming $m_{f}=0.01 \mathrm{GeV}$ for light quarks and $m_{c}=1.27 \mathrm{GeV}$ for the charm.

Finally, the differential cross section for the exclusive processes will be calculated including the corrections associated to the real part of the amplitude and the skewedness factor, which is related to the fact that the gluons emitted from the quark and antiquark into the dipole can carry different momentum fractions [57]. As a consequence,

$$
\frac{d \sigma^{\gamma^{*} p \rightarrow E p}}{d t}=\frac{R_{g}^{2}\left(1+\beta^{2}\right)}{16 \pi}\left|\mathcal{A}_{T, L}\left(x, Q^{2}, \boldsymbol{q}\right)\right|^{2},
$$

where $\beta$ is the ratio of real and imaginary parts of the amplitude, which will be estimated using that [31]

$$
\beta=\tan \left(\frac{\pi}{2} \bar{\delta}\right), \quad \text { with } \quad \bar{\delta}=\frac{\partial \ln \left(\operatorname{Im} \mathcal{A}_{T, L}\right)}{\partial \ln 1 / x} .
$$

Moreover, the skewedness factor $R_{g}^{2}$ will be calculated as follows [31]

$$
R_{g}=\frac{2^{2 \bar{\delta}+3}}{\sqrt{\pi}} \frac{\Gamma(\bar{\delta}+5 / 2)}{\Gamma(\bar{\delta}+4)},
$$

where $\bar{\delta}$ is defined as above. Adopting a given dipole amplitude and overlap functions, one can obtain the differential cross sections as a function of $W, Q^{2}$, and $t$. 
TABLE II. Parameters of the MPS model determined from fits to the reduced cross section from the combined HERA data in neutral current unpolarized $e p$ scattering and to the data for the exclusive $\rho$ and $J / \Psi$ production from H1 and ZEUS Collaborations. Results derived using the BG and LCG models for the vector meson wave functions.

\begin{tabular}{|c|c|c|c|c|c|c|c|}
\hline & $R_{p}\left(\mathrm{GeV}^{-1}\right)$ & $\gamma_{s}$ & $\lambda$ & $x_{0}$ & $c\left(\mathrm{GeV}^{-2}\right)$ & $B\left(\mathrm{GeV}^{-2}\right)$ & $\chi^{2} /$ d.o.f. \\
\hline BG & $3.44 \pm 0.06$ & $0.720 \pm 0.001$ & $0.207 \pm 0.028$ & $7.97 \times 10^{-6} \pm 0.03 \times 10^{-6}$ & $1.35 \pm 0.10$ & $3.25 \pm 0.02$ & 1.39 \\
\hline LCG & $3.46 \pm 0.03$ & $0.728 \pm 0.001$ & $0.205 \pm 0.008$ & $8.01 \times 10^{-6} \pm 0.03 \times 10^{-6}$ & $1.48 \pm 0.04$ & $3.09 \pm 0.54$ & 1.13 \\
\hline
\end{tabular}
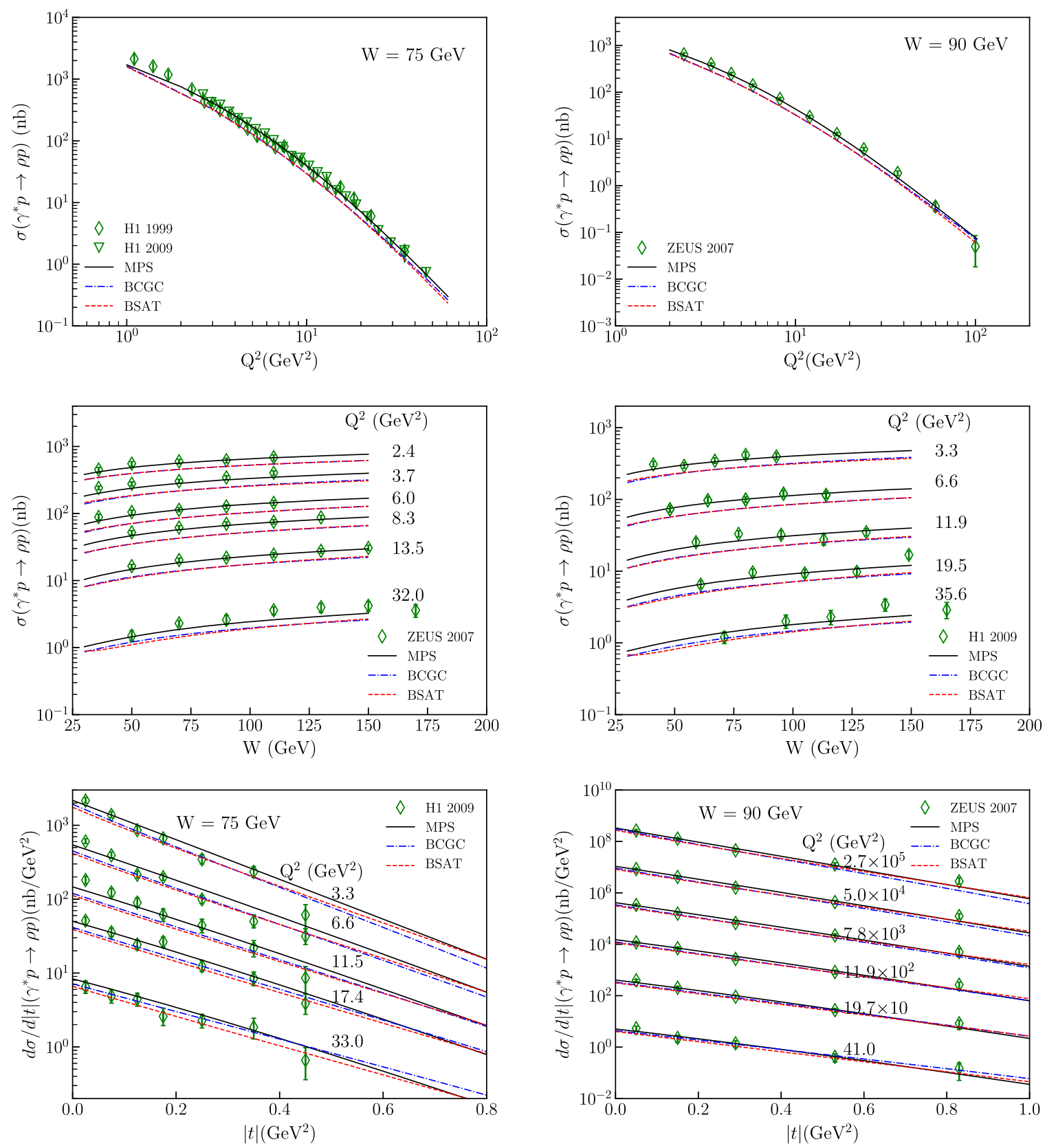

FIG. 2. Comparison of the MPS predictions with the experimental HERA data for the exclusive $\rho$ production. For completeness, the BSAT and BCGC results are also presented. Data from Refs. [60-62]. 
After integrating over $t$ the differential cross sections, one can obtain the total cross sections as a function of $W$ and $Q^{2}$.

\section{RESULTS}

First of all, we will update the MPS model considering the latest high precision combined HERA data for the reduced cross sections [58] as well as the H1 and ZEUS data for the exclusive $\rho$ and $J / \Psi$ production [59-64]. Such reanalysis of available data is motivated by the fact that a large amount of data, with extremely small error bars, were released after the study performed in Ref. [37]. We will include in our analysis the experimental data for the reduced cross section in the range $Q^{2} \in[0.045,45] \mathrm{GeV}^{2}$ and $x \leq 0.01$ and the statistical and systematic experimental uncertainties will be added in quadrature in the calculation of $\chi^{2}$. Moreover, we also will consider the experimental data for exclusive vector meson cross sections characterized by $Q^{2} \geq 1 \mathrm{GeV}^{2}$. We will consider the BG and LCG models for the vector meson wave functions and will use the data to fit the free parameters $c, B, R_{p}, \gamma_{s}, \lambda$, and $x_{0}$. The analysis of the MPS model will be performed assuming $m_{f}=0.14 \mathrm{GeV}$ for light quarks and $m_{c}=$ $1.4 \mathrm{GeV}$ for the charm quark.
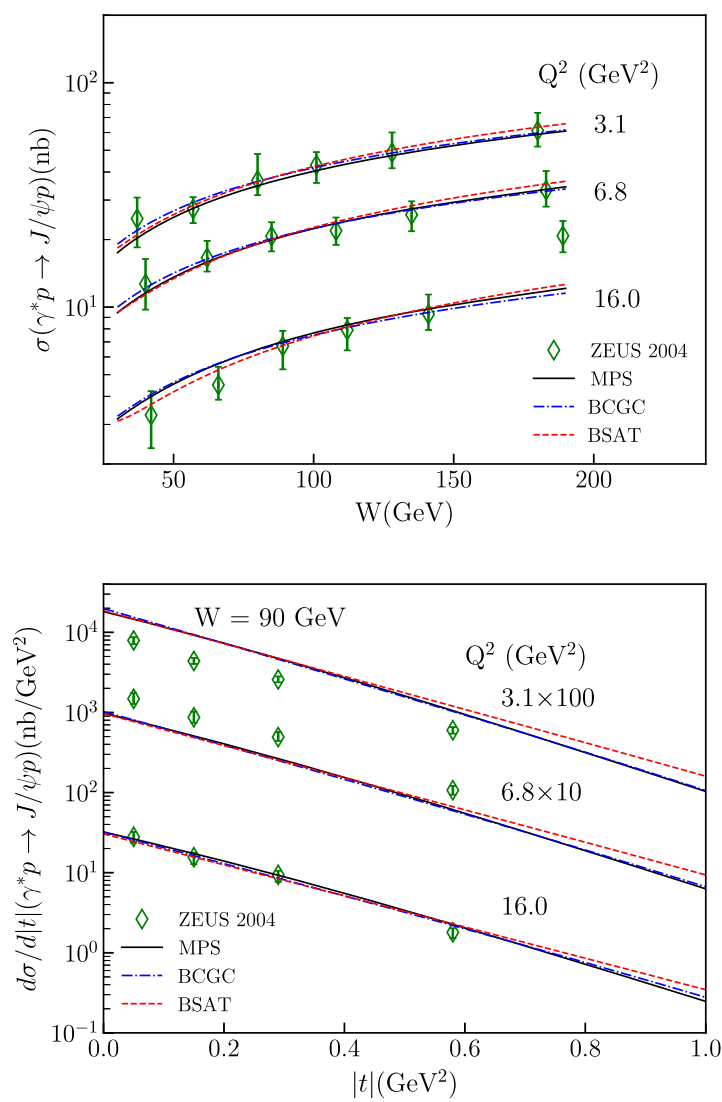

Our results are presented in Table II. The values of $R_{p}$, $\gamma_{s}, \lambda$, and $x_{0}$ are similar for the different models of wave functions, which is associated to the fact that they are mainly constrained by the data for the reduced cross section. In contrast, the $\chi^{2}$ results of the fit are strongly dependent of the models assumed to describe the vector meson wave functions. The smaller $\chi^{2}$ is obtained for the LCG model, with this value being smaller than those obtained in Refs. [37,55,56]. In what follows, we will restrict our analysis to the LCG model. It is important to emphasize that one also has considered the possibility that the mesons are described by distinct wave functions, as in Ref. [37], but the resulting $\chi^{2}$ is not better than the value obtained assuming the LCG model for both mesons.

In Figs. 2 and 3 we compare the MPS predictions with the experimental HERA data for the exclusive $\rho$ and $J / \Psi$ production used in the fitting procedure. For completeness, the BSAT and BCGC results are also presented. In the kinematical range covered by HERA, the predictions of the distinct saturation models for the $J / \Psi$ production are similar, with the main difference occurring in the behavior of $d \sigma / d t$ for $t \geq 0.4 \mathrm{GeV}^{2}$. In contrast, for the $\rho$ production, the MPS model predicts larger values for the total cross section and for the differential distribution at small $t$.
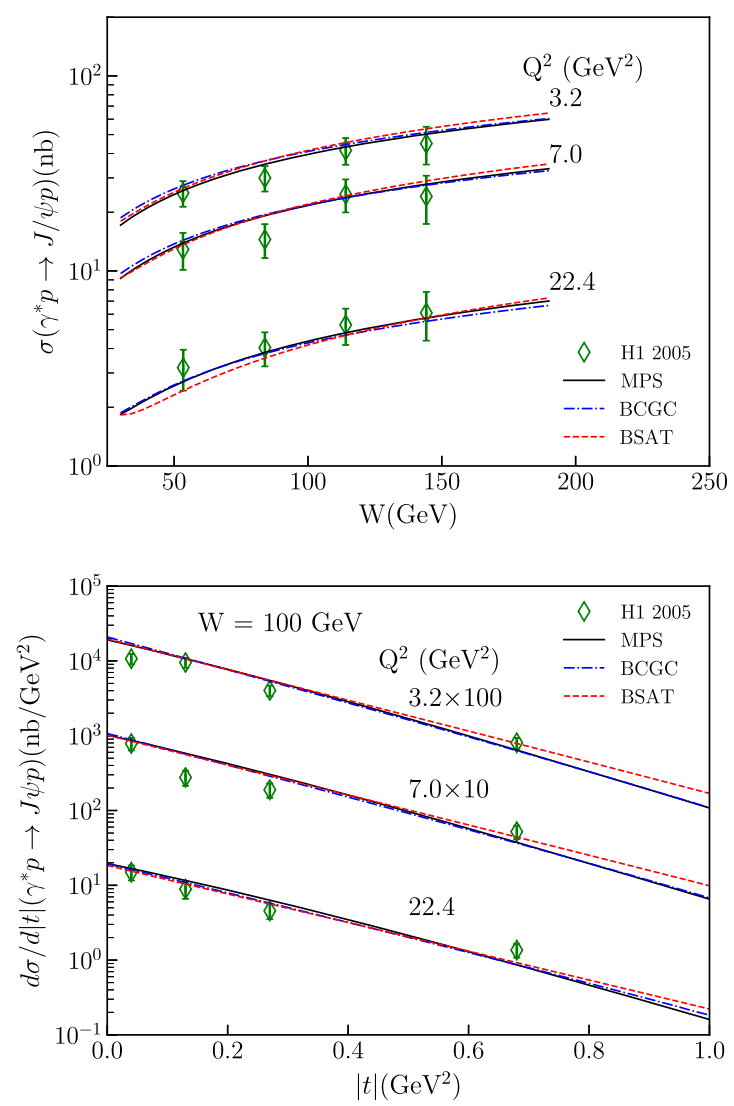

FIG. 3. Comparison of the MPS predictions with the experimental HERA data for the exclusive $J / \Psi$ production. For completeness, the BSAT and BCGC results are also presented. Data from Refs. [63,64]. 
In order to further test the MPS model, in Fig. 4 we confront experimental data from $\mathrm{H} 1$ and ZEUS Collaborations for the DVCS process with model predictions for photon virtuality, energy and $t$ dependencies. It is important to emphasize that these data were not included in the fit. The description of the data is quite good, with the MPS predictions being similar to those from the BSAT and BCGC models. As in the case of the vector meson production, the predictions of the distinct saturation models differ for $t \geq 0.4 \mathrm{GeV}^{2}$.
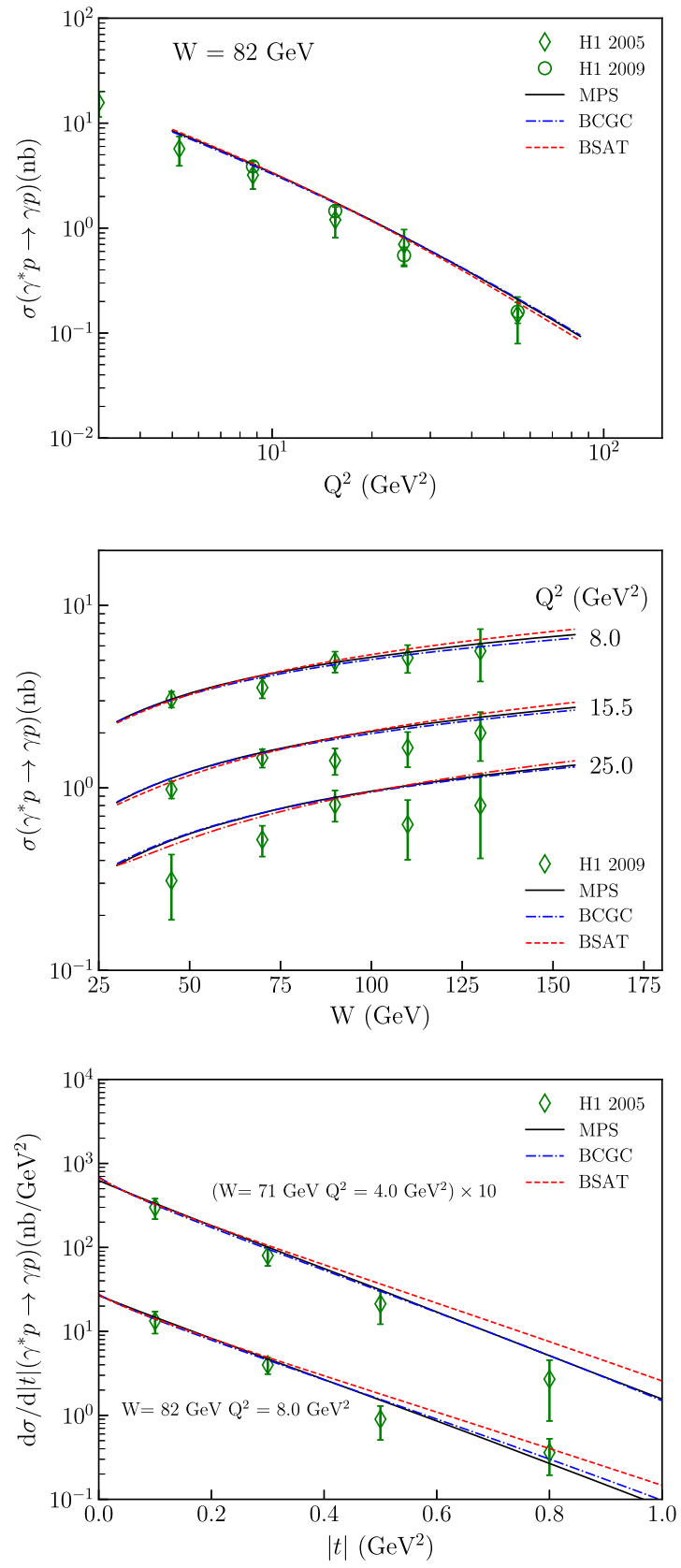

As discussed in Sec. I, the search for the saturation effects is one of the major motivations for the construction of the EIC in the USA [1], recently approved, as well as for the proposal of future electron-hadron colliders at CERN [2]. These colliders are expected to allow the investigation of the hadronic structure with unprecedented precision to inclusive and diffractive observables. In particular, the measurement of $d \sigma / d t$ with small error bars in a larger range of values for $t$ is expected to be feasible at the EIC and $\mathrm{LHeC}$. Moreover, these future colliders are expected to
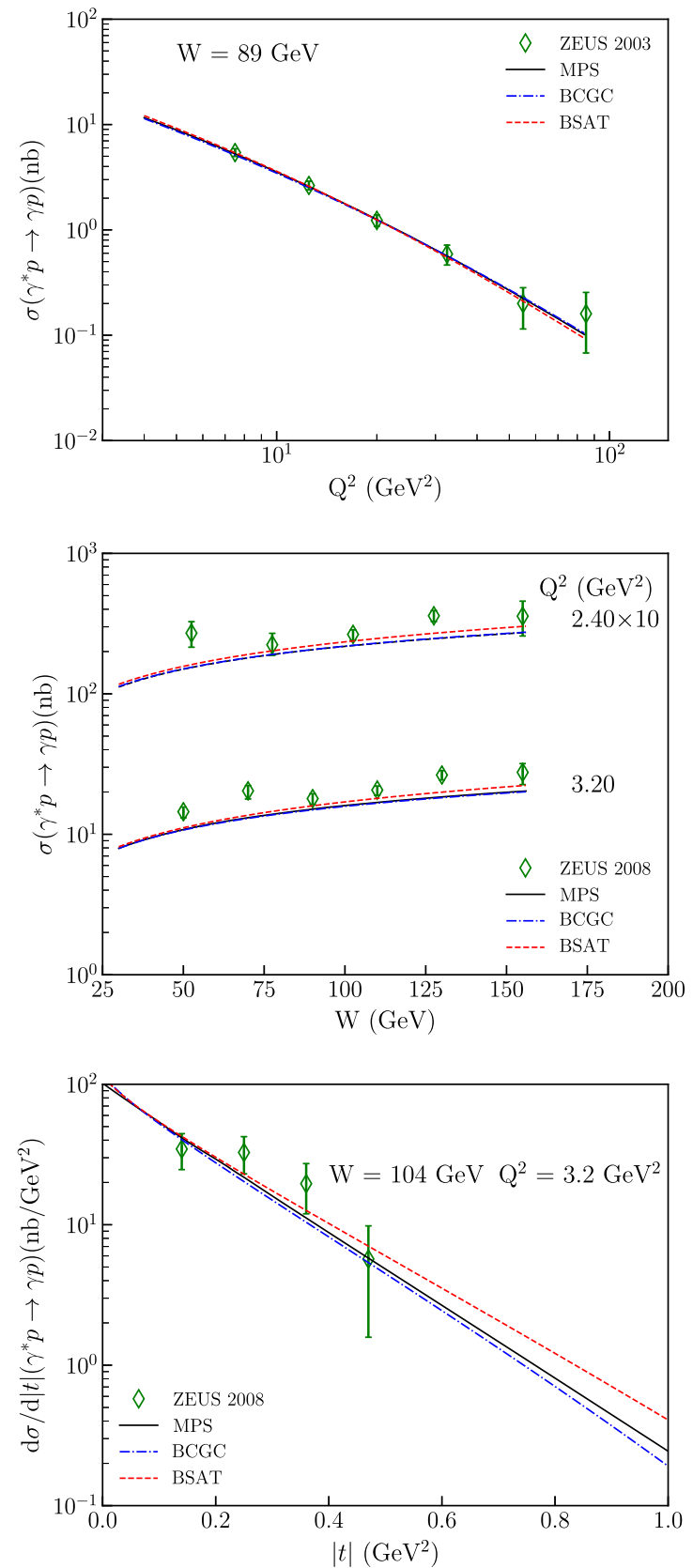

FIG. 4. Predictions of the MPS model for the DVCS process considering the kinematical range covered by HERA. For completeness, the BSAT and BCGC results are also presented. Data from H1 and ZEUS Collaborations [65-68]. 
be also able to separate the events in which the virtual photon has longitudinal or transverse polarization. Such aspects motivate the analysis of exclusive processes in the kinematical range that will be probed by the EIC and LHeC. In what follows we will perform a detailed comparison between the MPS, BSAT, and BCGC predictions, considering two values for the photon virtuality $\left(Q^{2}=2\right.$ and $\left.10 \mathrm{GeV}^{2}\right)$ and for $\gamma p$ center-of-mass energy ( $W=100$ and $1000 \mathrm{GeV}$ ). Moreover, we will present, for the first time, the predictions for the $t$ distributions considering the distinct photon polarizations. The predictions of the distinct saturation models for the energy dependence of the total cross sections are shown in Fig. 5. The MPS predictions for $\rho$ production are similar to those from the BSAT one for large $W$ and small $Q^{2}$. For $Q^{2}=10 \mathrm{GeV}^{2}$, the predictions of the MPS model become larger than those derived adopting the impact parameter saturation models. In contrast, for the $J / \Psi$ and DVCS cases, the MPS and BCGC
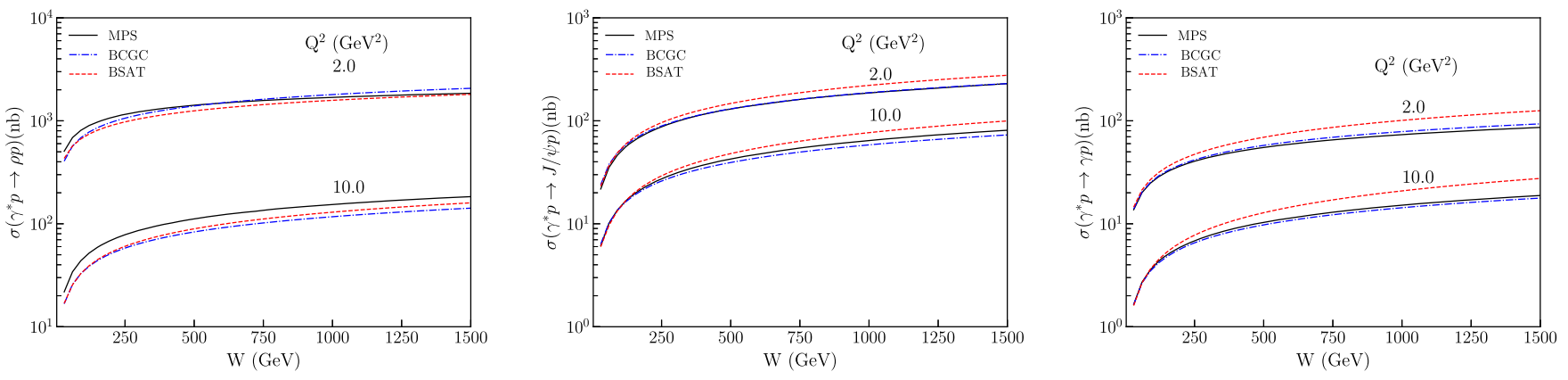

FIG. 5. Predictions of the distinct saturation models for the energy dependence of the $\rho$ (left panel), $J / \Psi$ (center panel), and DVCS (right panel) cross sections for two distinct values of $Q^{2}$.
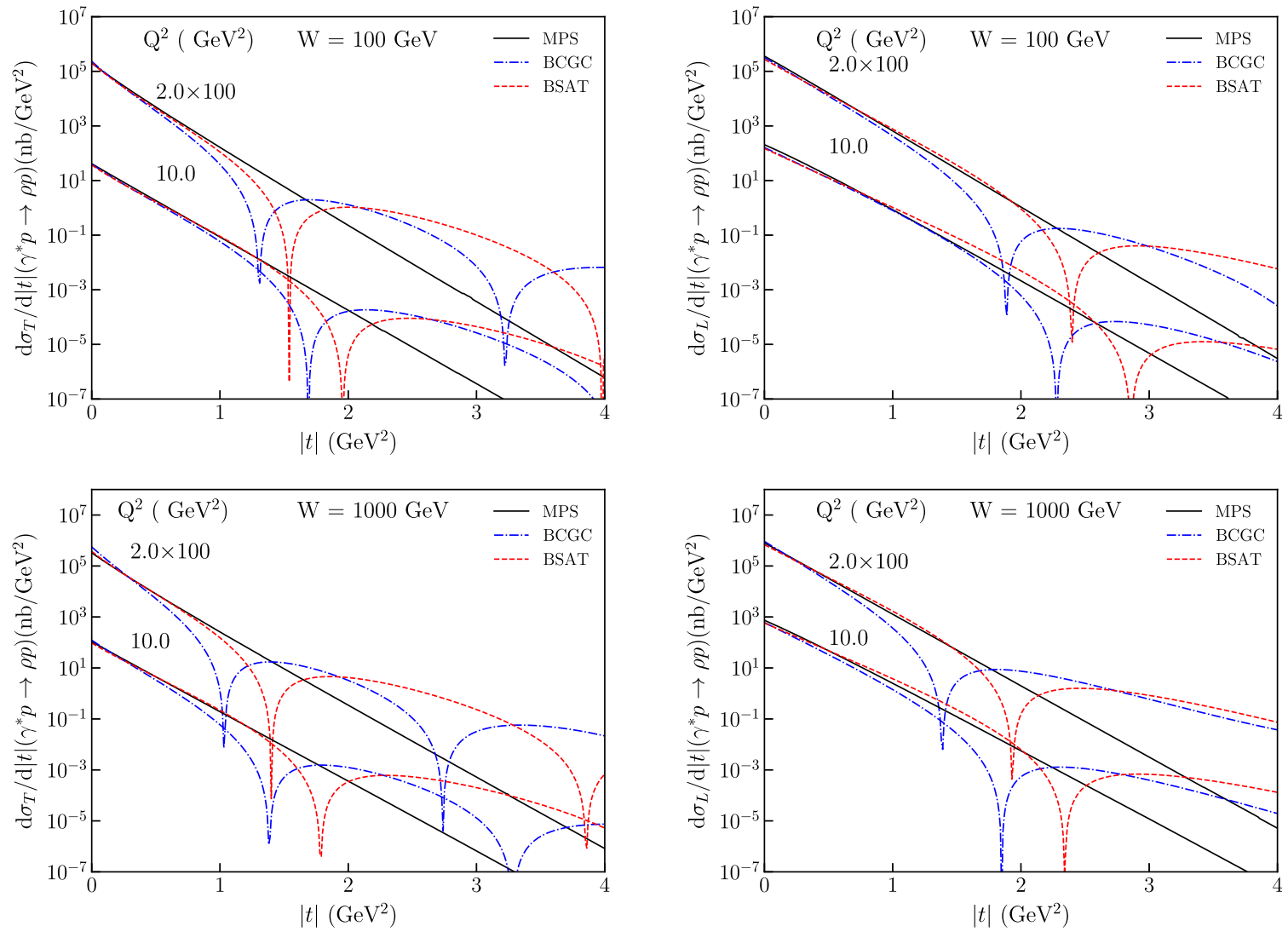

FIG. 6. Predictions of the different saturation models for $d \sigma / d t$ considering the exclusive $\rho$ production for distinct values of $Q^{2}$ and $W$ and that the virtual photon has a transverse (left panels) or longitudinal (right panels) polarization. Results for $W=100 \mathrm{GeV}$ $(1000 \mathrm{GeV})$ are presented in the upper (lower) panels. 
predictions are almost identical and are smaller than the BSAT results. Our results indicate that, in order to constrain the description of the QCD dynamics, less inclusive observables must be investigated.

In Figs. 6 and 7 we display our predictions for the exclusive $\rho$ and $J / \Psi$ production, respectively, considering that the virtual photon has a transverse (left panels) or longitudinal (right panels) polarization. Results for $W=$ $100 \mathrm{GeV}(1000 \mathrm{GeV})$ are presented in the upper (lower) panels. The distinct predictions are similar for $|t| \approx 0$, which is expected from the results obtained in Fig. 5. However, they significantly differ at large values of the squared transferred momentum. While the BSAT and BCGC models predict the presence of dips in the distribution, these are not predicted by the MPS model in the kinematical range considered, independent of the photon polarization. Such a result is directly associated to the factorization of the form factor $f(\boldsymbol{q})$ in the MPS model. In contrast, for the BSAT and BCGC models, the position of the dip is dependent of the dipole model, with the first dip predicted by the BCGC model occurring for smaller values of $|t|$ in comparison to the BSAT prediction. Moreover, the first dip also occurs for smaller values of $|t|$ when the photon virtuality is decreased or the center-of-mass energy
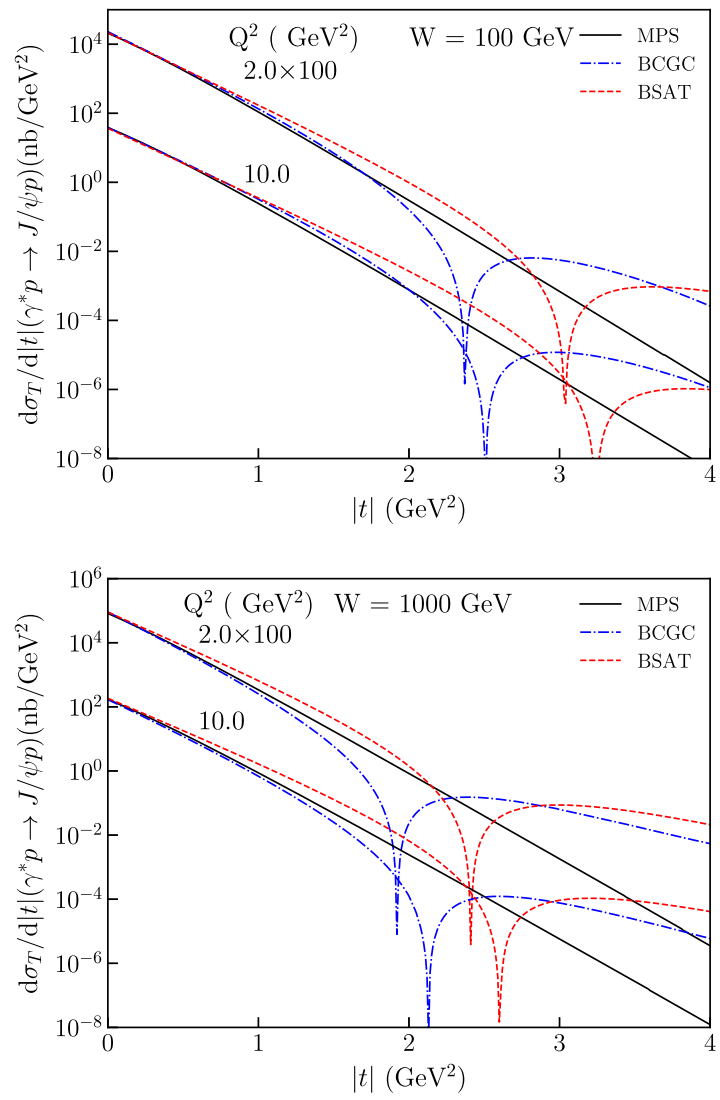

is increased. Another important aspect is that the position of the dips is dependent of the photon polarization, with the dip predicted by the BSAT and BCGC models occurring for larger values of $|t|$ in the case of a photon with a longitudinal polarization. In particular, for the $\rho$ production, we predict a large difference between the positions of the dips for longitudinal and transverse polarizations. Such result indicates that a future experimental analysis of $d \sigma / d t$ for the distinct photon polarizations will be useful to discriminate between the distinct phenomenological approaches for saturation physics.

In Fig. 8 we exhibit our predictions for $d \sigma / d t$ considering the sum over photon polarizations. Results for the exclusive $\rho$ (upper), $J / \Psi$ (middle), and $\gamma$ (lower) production are presented considering distinct values of $Q^{2}$ and $W$. When the contribution of both photon polarizations are added, the shape of the differential distributions for $\rho$ and $J / \Psi$ predicted by the BSAT and BCGC models are modified. In particular, for the $\rho$ production, where the difference between the position of the dips in the longitudinal and transverse polarizations is not negligible, the diffraction pattern predicted by these models almost vanishes. As a consequence, the predictions of the distinct saturation models become similar. For the $J / \Psi$ case, one
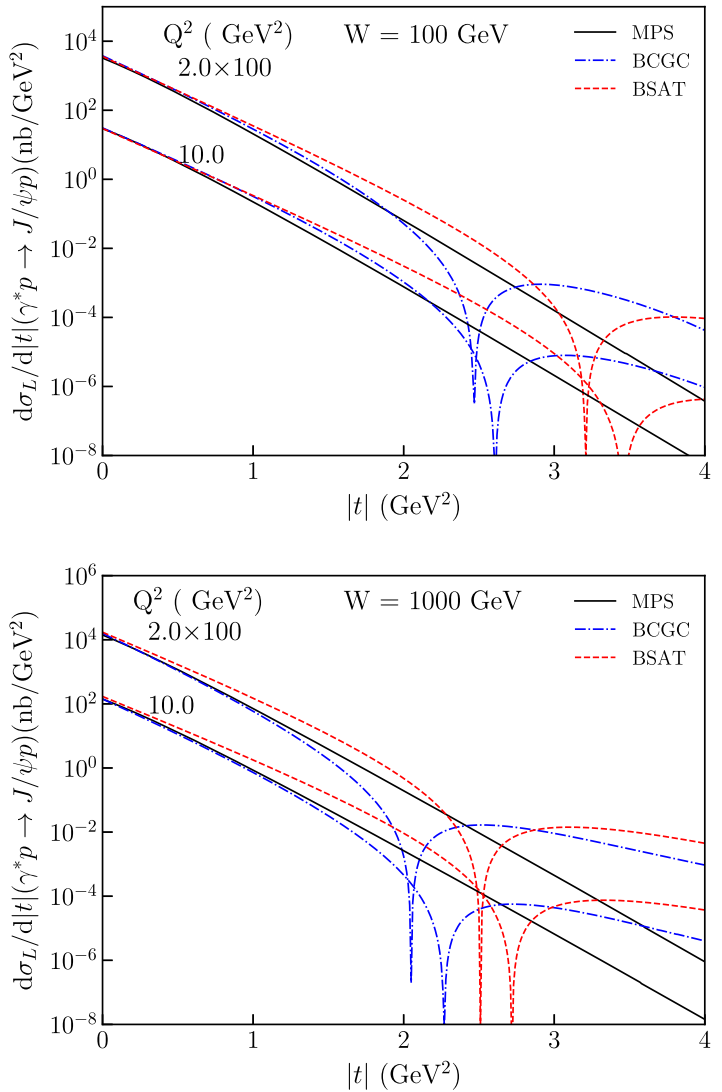

FIG. 7. Predictions of the different saturation models for $d \sigma / d t$ considering the exclusive $J / \Psi$ production for distinct values of $Q^{2}$ and $W$ and that the virtual photon has a transverse (left panels) or longitudinal (right panels) polarization. Results for $W=100 \mathrm{GeV}$ $(1000 \mathrm{GeV})$ are presented in the upper (lower) panels. 

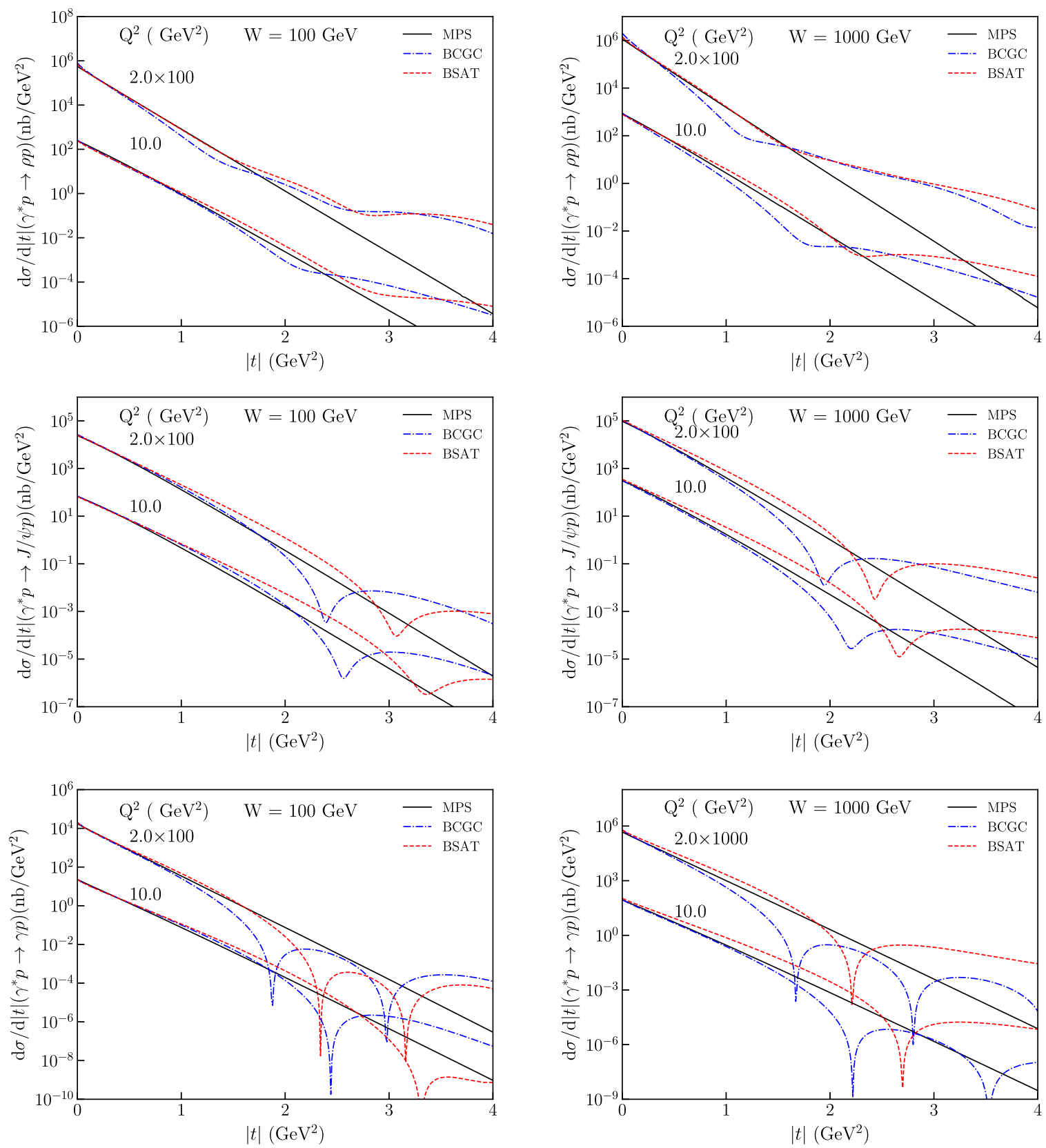

FIG. 8. Predictions of the different saturation models for $d \sigma / d t$, summed over photon polarizations, considering the exclusive $\rho$ (upper), $J / \Psi$ (middle), and $\gamma$ (lower) production for distinct values of $Q^{2}$ and $W$. Results for $W=100 \mathrm{GeV}(1000 \mathrm{GeV})$ are presented in the left (right) panels.

has the diffraction pattern is still present, which implies that the analysis of the total $d \sigma / d t$ is also a good observable to discriminate between the distinct approaches. Finally, for the DVCS process (lower panels), which is characterized by incoming photons of transverse polarization, the predictions of the different saturation models are very distinct, which indicate that a future experimental analysis of the exclusive photon production, considering events characterized by squared transferred momentum $|t|$ larger than $1.8 \mathrm{GeV}^{2}$, will be able to constrain the description of the QCD dynamics.

\section{SUMMARY}

During recent years, several studies have shown that the exclusive production of vector mesons and photons in $e p$ collisions have the potential to probe the QCD dynamics at high energies. In particular, there is the expectation that the large data sample to be obtained at the EIC and $\mathrm{LHeC}$ will allow a more detailed study of the exclusive cross sections and a better discrimination between alternative descriptions. In this paper we have presented a comprehensive study of the exclusive $\rho, J / \Psi$, and $\gamma$ production in $e p$ collisions at future colliders using the color dipole formalism. We have used 
three different models for the dipole scattering amplitude, which take into account the nonlinear (saturation) effects of the QCD dynamics. In particular, we have updated the MPS model, which is based on the solutions of the BK equation in the momentum space, and is characterized by a saturation scale that is dependent of the squared momentum transfer $t$ and by the factorization of the $t$ dependence associated to the proton vertex. A very good description of the current data is obtained when the light cone Gauss model is assumed for the vector meson wave functions. A detailed comparison of the MPS predictions with the BSAT and BCGC results is performed. Differently from the BSAT and BCGC model, a diffractive pattern in $d \sigma / d t$ is not predicted by the MPS model. Moreover, the BSAT and BCGC predictions for the position of the dips are distinct and dependent on the virtual photon polarization. Our results indicate that a future experimental analysis of exclusive processes, considering events characterized by large values of the squared transferred momentum, has the potentiality of constraining the description of the QCD dynamics.

\section{ACKNOWLEDGMENTS}

The work is partially supported by the Strategic Priority Research Program of Chinese Academy of Sciences (Grant No. XDB34030301). V. P. G. was partially financed by the Brazilian funding agencies CNPq, FAPERGS, and INCTFNA (Grant No. 464898/2014-5).
[1] D. Boer, M. Diehl, R. Milner, R. Venugopalan, W. Vogelsang, D. Kaplan, H. Montgomery, S. Vigdor et al., arXiv: 1108.1713; A. Accardi, J. L. Albacete, M. Anselmino, N. Armesto, E. C. Aschenauer, A. Bacchetta, D. Boer, W. Brooks et al., Eur. Phys. J. A 52, 268 (2016); E. C. Aschenauer, S. Fazio, J. H. Lee, H. Mäntysaari, B. S. Page, B. Schenke, T. Ullrich, R. Venugopalan, and P. Zurita, Rep. Prog. Phys. 82, 024301 (2019).

[2] J. L. Abelleira Fernandez et al. (LHeC Study Group), J. Phys. G 39, 075001 (2012); P. Agostini et al., J. Phys. G 2021) 110501,48$)$.

[3] M. Klein and R. Yoshida, Prog. Part. Nucl. Phys. 61, 343 (2008).

[4] F. Gelis, E. Iancu, J. Jalilian-Marian, and R. Venugopalan, Annu. Rev. Nucl. Part. Sci. 60, 463 (2010); H. Weigert, Prog. Part. Nucl. Phys. 55, 461 (2005); J. Jalilian-Marian and Y. V. Kovchegov, Prog. Part. Nucl. Phys. 56, 104 (2006).

[5] E. R. Cazaroto, F. Carvalho, V. P. Goncalves, and F. S. Navarra, Phys. Lett. B 671, 233 (2009).

[6] V. P. Goncalves, M. S. Kugeratski, M. V. T. Machado, and F. S. Navarra, Phys. Rev. C 80, 025202 (2009).

[7] A. Caldwell and H. Kowalski, Phys. Rev. C 81, 025203 (2010).

[8] T. Lappi and H. Mantysaari, Phys. Rev. C 83, 065202 (2011).

[9] T. Toll and T. Ullrich, Phys. Rev. C 87, 024913 (2013).

[10] N. Armesto and A. H. Rezaeian, Phys. Rev. D 90, 054003 (2014).

[11] V. P. Gonçalves and D. S. Pires, Phys. Rev. C 91, 055207 (2015).

[12] T. Lappi, H. Mantysaari, and R. Venugopalan, Phys. Rev. Lett. 114, 082301 (2015).

[13] H. Mantysaari and B. Schenke, Phys. Rev. Lett. 117, 052301 (2016).

[14] H. Mantysaari and B. Schenke, Phys. Rev. D 94, 034042 (2016).
[15] V. P. Goncalves, F. S. Navarra, and D. Spiering, Phys. Lett. B 768, 299 (2017).

[16] J. Cepila, J. G. Contreras, and M. Krelina, Phys. Rev. C 97, 024901 (2018).

[17] A. Luszczak and W. Schafer, Phys. Rev. C 97, 024903 (2018).

[18] H. Mantysaari and R. Venugopalan, Phys. Lett. B 781, 664 (2018).

[19] V. P. Goncalves, F. S. Navarra, and D. Spiering, Phys. Lett. B 791, 299 (2019).

[20] D. Bendova, J. Cepila, and J. G. Contreras, Phys. Rev. D 99, 034025 (2019).

[21] M. Krelina, V. P. Goncalves, and J. Cepila, Nucl. Phys. A989, 187 (2019).

[22] M. Lomnitz and S. Klein, Phys. Rev. C 99, 015203 (2019).

[23] H. Mantysaari and B. Schenke, Phys. Rev. C 101, 015203 (2020).

[24] Y. Hatta, B. W. Xiao, and F. Yuan, Phys. Rev. D 95, 114026 (2017).

[25] V. P. Goncalves, D. E. Martins, and C. R. Sena, Nucl. Phys. A1004, 122055 (2020).

[26] H. Mäntysaari, K. Roy, F. Salazar, and B. Schenke, Phys. Rev. D 103, 094026 (2021).

[27] D. Bendova, J. Cepila, J. G. Contreras, V. P. Gonçalves, and M. Matas, Eur. Phys. J. C 81, 211 (2021).

[28] H. Mäntysaari, Rep. Prog. Phys. 83, 082201 (2020).

[29] N. N. Nikolaev and B. G. Zakharov, Z. Phys. C 49, 607 (1991); 53, 331 (1992).

[30] H. Kowalski and D. Teaney, Phys. Rev. D 68, 114005 (2003).

[31] H. Kowalski, L. Motyka, and G. Watt, Phys. Rev. D 74, 074016 (2006).

[32] G. Watt and H. Kowalski, Phys. Rev. D 78, 014016 (2008).

[33] J. Cepila, J. G. Contreras, and M. Matas, Phys. Rev. D 99, 051502 (2019).

[34] D. Bendova, J. Cepila, J. G. Contreras, and M. Matas, Phys. Rev. D 100, 054015 (2019). 
[35] J. Jalilian-Marian, A. Kovner, L. McLerran, and H. Weigert, Phys. Rev. D 55, 5414 (1997); J. Jalilian-Marian, A. Kovner, and H. Weigert, Phys. Rev. D 59, 014014 (1998); 59, 014015 (1998); 59, 034007 (1999); A. Kovner, J. Guilherme Milhano, and H. Weigert, Phys. Rev. D 62, 114005 (2000); H. Weigert, Nucl. Phys. A703, 823 (2002); E. Iancu, A. Leonidov, and L. McLerran, Nucl. Phys. A692, 583 (2001); E. Ferreiro, E. Iancu, A. Leonidov, and L. McLerran, Nucl. Phys. 703, 489 (2002).

[36] C. Contreras, E. Levin, R. Meneses, and I. Potashnikova, Phys. Rev. D 94, 114028 (2016); Eur. Phys. J. C 78, 475 (2018).

[37] C. Marquet, R. B. Peschanski, and G. Soyez, Phys. Rev. D 76, 034011 (2007).

[38] C. Marquet, R. B. Peschanski, and G. Soyez, Nucl. Phys. A756, 399 (2005).

[39] C. Marquet and G. Soyez, Nucl. Phys. A760, 208 (2005).

[40] I. I. Balitsky, Nucl. Phys. B463, 99 (1996); Y. V. Kovchegov, Phys. Rev. D 60, 034008 (1999).

[41] T. Lappi, H. Mäntysaari, and J. Penttala, Phys. Rev. D 102, 054020 (2020).

[42] G. Beuf, H. Hänninen, T. Lappi, and H. Mäntysaari, Phys. Rev. D 102, 074028 (2020).

[43] H. Mäntysaari and J. Penttala, Phys. Lett. B 823, 136723 (2021).

[44] J. Nemchik, N. N. Nikolaev, E. Predazzi, and B. G. Zakharov, Z. Phys. C 75, 71 (1997).

[45] H. G. Dosch, T. Gousset, G. Kulzinger, and H. J. Pirner, Phys. Rev. D 55, 2602 (1997); G. Kulzinger, H. G. Dosch, and H. J. Pirner, Eur. Phys. J. C 7, 73 (1999).

[46] J. R. Forshaw, R. Sandapen, and G. Shaw, Phys. Rev. D 69 , 094013 (2004).

[47] V. P. Goncalves and M. V. T. Machado, Eur. Phys. J. C 38, 319 (2004).

[48] J. L. Albacete, N. Armesto, J. G. Milhano, P. Quiroga-Arias, and C. A. Salgado, Eur. Phys. J. C 71, 1705 (2011).

[49] R. A. Fisher, Ann. Eugenics 7, 355 (1937); A. Kolmogorov, I. Petrovsky, and N. Piscounov, Moscou Univ. Bull. Math. A1, 1 (1937).
[50] S. Munier and R. B. Peschanski, Phys. Rev. Lett. 91, 232001 (2003); Phys. Rev. D 69, 034008 (2004); 70, 077503 (2004).

[51] A. M. Stasto, K. J. Golec-Biernat, and J. Kwiecinski, Phys. Rev. Lett. 86, 596 (2001).

[52] V. P. Goncalves and M. V. T. Machado, Phys. Rev. Lett. 91, 202002 (2003).

[53] C. Marquet and L. Schoeffel, Phys. Lett. B 639, 471 (2006).

[54] E. Iancu, K. Itakura, and S. Munier, Phys. Lett. B 590, 199 (2004).

[55] A. H. Rezaeian, M. Siddikov, M. Van de Klundert, and R. Venugopalan, Phys. Rev. D 87, 034002 (2013).

[56] A. H. Rezaeian and I. Schmidt, Phys. Rev. D 88, 074016 (2013).

[57] A. G. Shuvaev, K. J. Golec-Biernat, A. D. Martin, and M. G. Ryskin, Phys. Rev. D 60, 014015 (1999).

[58] H. Abramowicz et al. (H1 and ZEUS Collaborations), Eur. Phys. J. C 75, 580 (2015).

[59] J. Breitweg et al. (ZEUS Collaboration), Eur. Phys. J. C 6, 603 (1999).

[60] C. Adloff et al. (H1 Collaboration), Eur. Phys. J. C 13, 371 (2000).

[61] S. Chekanov et al. (ZEUS Collaboration), PMC Phys. A 1, 6 (2007).

[62] F. D. Aaron et al. (H1 Collaboration), J. High Energy Phys. 05 (2010) 032.

[63] A. Aktas et al. (H1 Collaboration), Eur. Phys. J. C 46, 585 (2006).

[64] S. Chekanov et al. (ZEUS Collaboration), Nucl. Phys. B695, 3 (2004).

[65] A. Aktas et al. (H1 Collaboration), Eur. Phys. J. C 44, 1 (2005).

[66] S. Chekanov et al. (ZEUS Collaboration), Phys. Lett. B 573, 46 (2003).

[67] S. Chekanov et al. (ZEUS Collaboration), J. High Energy Phys. 05 (2009) 108.

[68] F. D. Aaron et al. (H1 Collaboration), Phys. Lett. B 681, 391 (2009). 